\title{
ARTICLE
}

Received 4 May 2015 | Accepted 21 Jul 2015 | Published 18 Sep 2015

DOI: $10.1038 /$ ncomms 9121

OPEN

\section{Atomic-like spin noise in solid-state demonstrated with manganese in cadmium telluride}

\author{
S. Cronenberger ${ }^{1}$, D. Scalbert ${ }^{1}$, D. Ferrand ${ }^{2,3}$, H. Boukari ${ }^{2,3} \&$ J. Cibert ${ }^{2,3}$
}

Spin noise spectroscopy is an optical technique which can probe spin resonances non-perturbatively. First applied to atomic vapours, it revealed detailed information about nuclear magnetism and the hyperfine interaction. In solids, this approach has been limited to carriers in semiconductor heterostructures. Here we show that atomic-like spin fluctuations of $\mathrm{Mn}$ ions diluted in CdTe (bulk and quantum wells) can be detected through the Kerr rotation associated to excitonic transitions. Zeeman transitions within and between hyperfine multiplets are clearly observed in zero and small magnetic fields and reveal the local symmetry because of crystal field and strain. The linewidths of these resonances are close to the dipolar limit. The sensitivity is high enough to open the way towards the detection of a few spins in systems where the decoherence due to nuclear spins can be suppressed by isotopic enrichment, and towards spin resonance microscopy with important applications in biology and materials science.

\footnotetext{
${ }^{1}$ Laboratoire Charles Coulomb UMR 5221 CNRS/UM2, Université Montpellier, Place Eugene Bataillon, Montpellier Cedex 05 34095, France. ${ }^{2}$ Université Grenoble Alpes, Grenoble F-38000, France. ${ }^{3}$ CNRS, Institut NEEL, Grenoble F-38000, France. Correspondence and requests for materials should be addressed to D.S. (email: Denis.Scalbert@univ-montp2.fr).
} 
C oupling polarized light to atomic spin ensembles non resonantly, the atom-light interface ${ }^{1,2}$, is a powerful approach to manipulate collective spin states, and to generate spin squeezed ${ }^{3,4}$ and entangled atomic states ${ }^{5}$. Atomic ensembles, with which these methods have been developed, are hardly scalable. Scalability is more straightforwardly achieved in solids, however such an approach has been limited to carriers in semiconductor heterostructures ${ }^{6-12}$.

Here, we introduce the atom-exciton-light interface in solids, to transfer the atomic spin noise to light polarization in a system where the atomic spin is not directly coupled to light: in the case of $\mathrm{Mn}$ in $\mathrm{CdTe}$ which we use as a testbed, the exciton mediates the coupling between atoms and light. This interface is based on the sp-d exchange interaction, which couples the manganese ions to the carriers. Thus atomic spin fluctuations produce tiny splittings of the excitonic transitions, ultimately detected by the induced circular birefringence (Kerr rotation). We show that the spin noise spectra keep their atomic nature despite the strong hybridization between the orbitals of manganese ion and the crystal. This opens new possibilities by transposing in solids the method of quantum physics, based on the atom-light interface.

\section{Results}

Spin Hamiltonian and the spin noise spectra. Electron spin resonances can be described by a spin Hamiltonian, in which the allowed terms are dictated by symmetry considerations. For Mn in a tetrahedral crystal field it takes the form ${ }^{13}$

$$
\begin{aligned}
H= & A \mathbf{I} \cdot \mathbf{S}+g \mu_{\mathrm{B}} \mathbf{B} \cdot \mathbf{S} \\
& +\frac{a}{6}\left[S_{\mathrm{x}}^{4}+S_{\mathrm{y}}^{4}+S_{\mathrm{z}}^{4}-\frac{1}{5} S(S+1)\left(3 S^{2}+3 S-1\right)\right]
\end{aligned}
$$

Here $\mathbf{I}$ and $\mathbf{S}$ are the $\mathrm{Mn}$ nuclear and electronic spins respectively, $\mu_{\mathrm{B}}$ is the Bohr magneton and $\mathbf{B}$ is the magnetic field. The Hamiltonian is composed of the hyperfine term, with
$A=-170.5 \mathrm{MHz}$, the Zeeman term, with $g=2$ the Mn $g$-factor, and the cubic crystal field term, with $a=89.5 \mathrm{MHz}$ (ref. 14).

The ${ }^{6} S_{5 / 2}$ Mn ground state is split by the hyperfine coupling in six F-levels (Fig. 1a). Without crystal field, the F-levels are split by a magnetic field into $2 F+1$ Zeeman levels, with a common $g$-factor $g_{F}=1$ because $S=I$. The crystal field further splits and mixes the hyperfine levels, which considerably increases the number of allowed transitions ${ }^{13}$.

According to the fluctuation-dissipation theorem the spectrum of the spin fluctuations for a single $\mathrm{Mn}$ spin in the direction of the unit vector $\hat{\alpha}$, at thermal equilibrium, is related to the susceptibility and is given by ${ }^{15}$

$$
(\mathbf{S} \cdot \hat{\alpha})_{\omega}^{2}=\sum_{n, m}\left(\rho_{n}+\rho_{m}\right)|\langle n|\mathbf{S} \cdot \hat{\alpha}| m\rangle|^{2} \frac{\gamma}{\left(\omega+\omega_{n m}\right)^{2}+\gamma^{2}}
$$

where the double summation is over the $(2 S+1) \times(2 I+1)=36$ eigenstates of the spin hamiltonian, $\rho_{n}$ is the occupation factor at thermal equilibrium of level $n$ with eigenfrequency $\omega_{n}$ and $\omega_{n m}=\omega_{m}-\omega_{n}$. For simplicity, we assume Lorentzian lines with a broadening parameter $\gamma$, common to all transitions, and written as $\gamma_{\mathrm{T}}$ for the transverse configuration $(\mathbf{B} \perp \hat{\alpha})$ and $\gamma_{\mathrm{L}}$ for the longitudinal geometry $(\mathbf{B} / / \hat{\alpha})$.

In the transverse configuration, Zeeman transitions within each hyperfine level contribute to a single line at $g_{F}=1$, and interhyperfine transitions between adjacent $F$-levels contribute to higher frequency lines. The sum rules derived in Supplementary Note 1 shows that half of the integrated spin noise is concentrated in the $g_{F}=1$ line, the other half being shared between all interhyperfine transitions. In the longitudinal case, $\mathbf{S} \cdot \hat{\alpha}$ is diagonal within each hyperfine level and the corresponding noise signal appears around zero frequency. The crystal field brings in some complexity but does not affect significantly the intensity share between intra- and inter-hyperfine transitions.
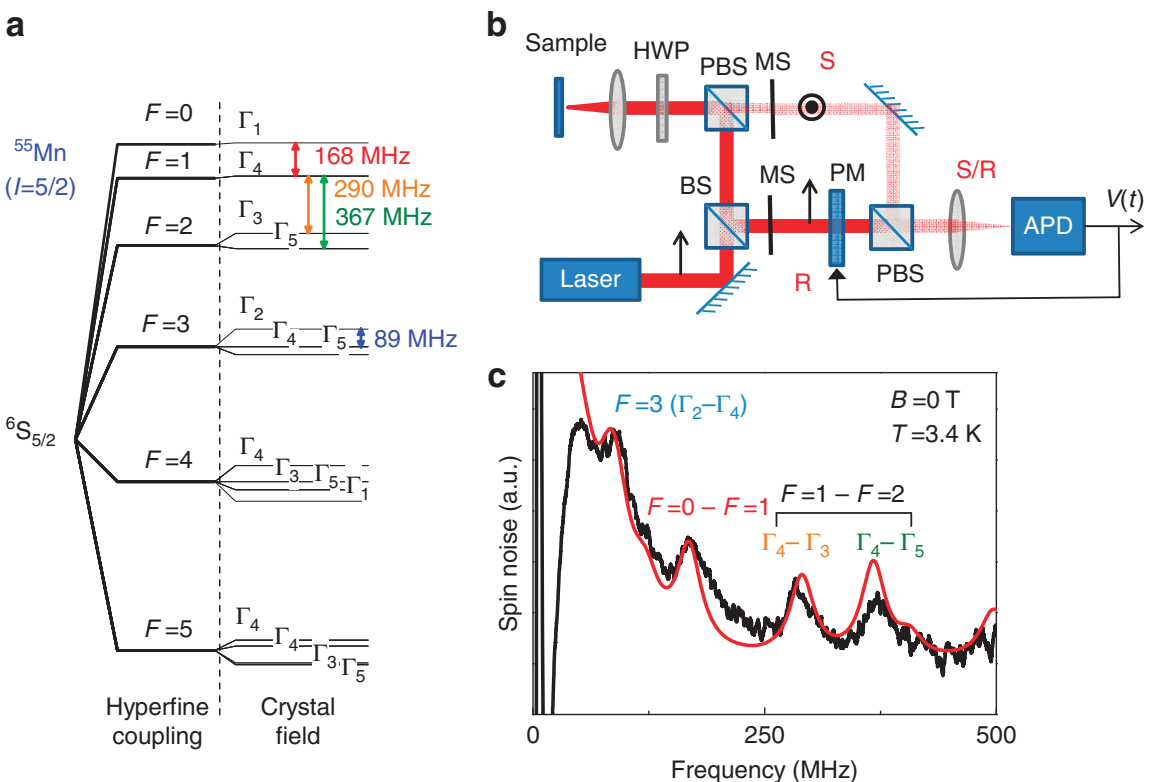

Figure 1 | Spin noise of ${ }^{\mathbf{5 5}} \mathbf{M n}$ diluted in bulk CdTe. (a) Energy levels of ${ }^{55} \mathrm{Mn}$ split by the hyperfine coupling and the cubic crystal field. Arrows point the transitions observed in c. (b) Experimental setup. A vertically polarized laser beam is focused on the sample. Mn spin fluctuations impart Kerr rotation noise on the reflected probe which is split into two mutually orthogonal polarizations (beam $S$ and $R$ ) by a polarizing beamsplitter (PBS). The beam $S$ carries the spin fluctuations while beam R carries only the intensity fluctuations. Both beams are sent alternatively on the avalanche diode (APD) using mechanical shutters (MS), and kept at the same intensity with a feedback loop on the phase modulator (PM). (c) Spin noise spectra in zero magnetic field, as measured (black line) and calculated (red line) with only one parameter $\gamma=100 \mathrm{MHz}$. The dip in the experimental spectrum below $50 \mathrm{MHz}$ comes from subtraction of a zero frequency line not accounted for by theory (see text). 


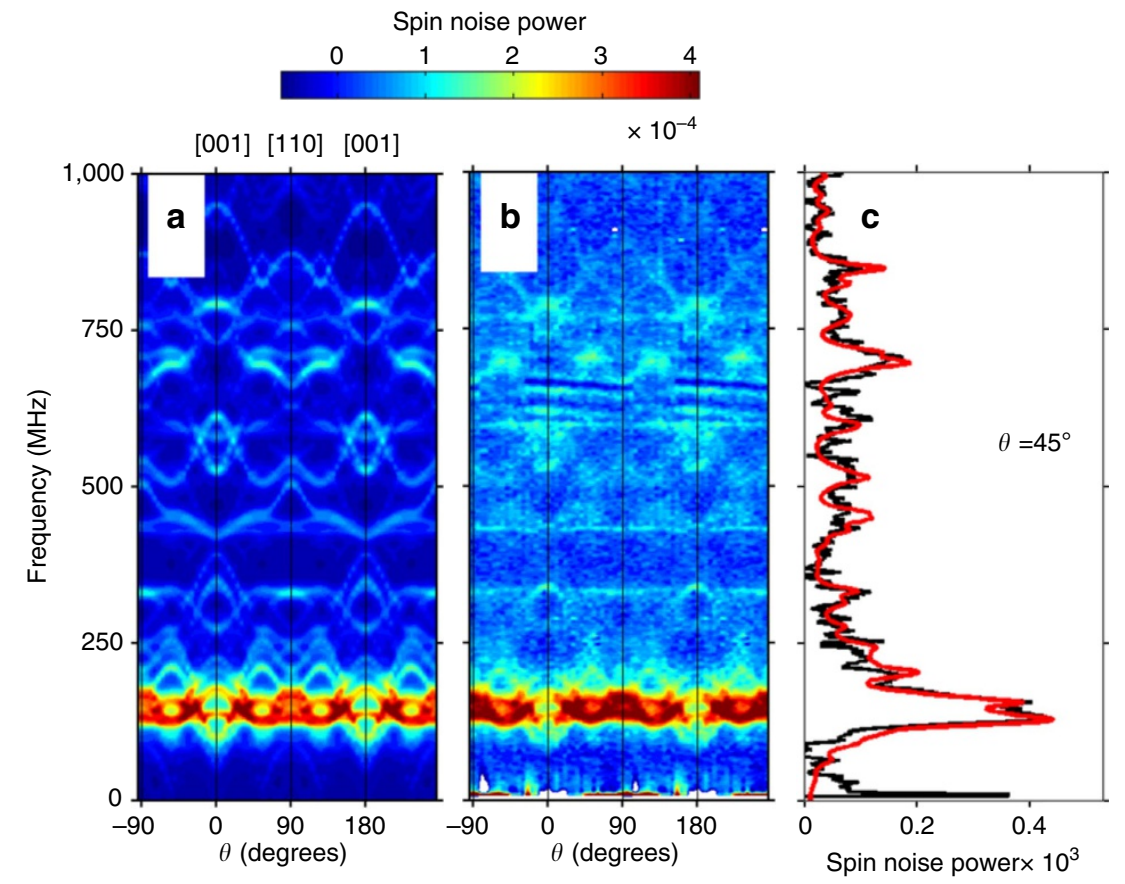

Figure 2 | Angular resolved spin noise of ${ }^{55} \mathbf{M n}$ diluted in bulk CdTe at $\boldsymbol{B}_{\mathbf{T}}=\mathbf{1 0 . 5} \mathbf{~ m T}$. (a) Contour plot of the spectra calculated with Equation (2) versus $\theta$, the angle between magnetic field and the [001] direction contained in the (110) plane. (b) Experimental contour plot $(T=4.8 \mathrm{~K})$. (c) Experimental spectrum at $\theta=45^{\circ}$ (black line) and best fit (red line) with $\gamma_{T}=50 \mathrm{MHz}$.

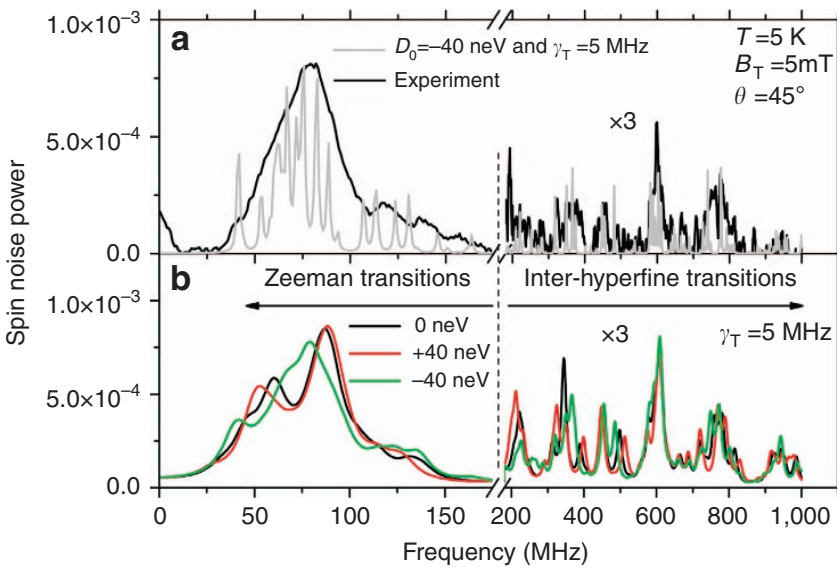

Figure 3 | Adjustment of the biaxial strain to fit the experimental spin noise spectra of ${ }^{55} \mathbf{M n}$ in the QW grown on CdTe. (a) Experimental (black line) and calculated (grey line) spin noise spectra. As shown by the calculation with a small broadening $\gamma_{T}=5 \mathrm{MHz}$, many unresolved individual lines, which belong either to the Zeeman transitions (left) or to inter-hyperfine transitions (right), are unresolved in the experimental spectrum. (b) Calculated noise spectra for different values of the strain parameter $D_{0}$ : the best agreement with the experiment is obtained for $D_{0}=-40 \mathrm{neV}$ and $\gamma_{T}=50 \mathrm{MHz}$ (green curve).

Samples and experiment. All the results are obtained from $\mathrm{Cd}_{0.999} \mathrm{Mn}_{0.001}$ Te samples: a bulk crystal cleaved along a (110) plane and quantum wells (QW) grown either on the (001) plane of $\mathrm{CdTe}$ or $\mathrm{Cd}_{96} \mathrm{Zn}_{0.04} \mathrm{Te}$ substrates. The spin fluctuations are probed along the laser beam, perpendicular to the sample surface. More details about the samples and the experimental setup (Fig. 1b) can be found in section Methods.

Bulk sample. The spin noise spectrum of Mn diluted in the bulk crystal is reported in Fig. 1c. The four strongest resonances are identified as intra-hyperfine, and inter-hyperfine transitions between states of the Mn split by the cubic crystal field (Fig. 1a). The data are well reproduced by the model with as only fitting parameter the HWHM in omega units $\gamma=100 \mathrm{MHz}$. Spin noise spectra have also been measured with a magnetic field applied in all directions of the (110) plane defined by the angle $\theta$ (by steps of $5^{\circ}$ ). As expected, we obtain a twofold symmetry in the frequency map. We then average the noise spectra measured at $\theta$ and $\theta+180^{\circ}$ in order to better resolve the weak structures, which spread over the $1 \mathrm{GHz}$ width (Fig. 2b,c). All the dominant features predicted by equation (2) can be recognized in the experimental spectra and many details are perfectly reproduced by the simulation with the linewidth $\gamma_{\mathrm{T}}$ being the only fitting parameter. A line centred at zero frequency, not predicted by the theory, has been subtracted from the experimental spectra to allow the comparison with theory: the original spectra are given in Supplementary Fig. 1.

Quantum wells. The spin noise spectra are quite sensitive to small lattice distortions. The presence of a lattice mismatch between the QW and substrate imposes adding a spin anisotropy term $D_{0} S_{\mathrm{z}}^{2}$ to the spin hamiltonian. This term is clearly observed in the QW grown on $\mathrm{Cd}_{96} \mathrm{Zn}_{0.04} \mathrm{Te}$, with the expected value $D_{0}=+473 \mathrm{neV}$ obtained using the known $\mathrm{Mn}$ spin-lattice coefficients $^{14}$. However, the fourfold cubic symmetry is broken. Adding an in-plane anisotropy term partially reproduces the experimental spectra (see Supplementary Fig. 2).

Including a $D_{0} S_{z}^{2}$ term also in the quasi-lattice-matched QW grown on CdTe is sufficient to obtain a relatively good agreement between experiment and theory (see Fig. 3). The anisotropy coefficient $D_{0}=-40 \mathrm{neV}$ is significantly larger than the calculated value $D_{0}=-5 \mathrm{neV}$. The expected fourfold cubic symmetry for magnetic fields applied in the (001) plane of the QW is observed (Fig. 4). Fig. 3a also reveals that the line centred at $75 \mathrm{MHz}$ consists in many unresolved individual lines. Fig. 5 shows that this bunch of lines shifts almost linearly with the magnetic field, and can be assigned to the Zeeman transitions 


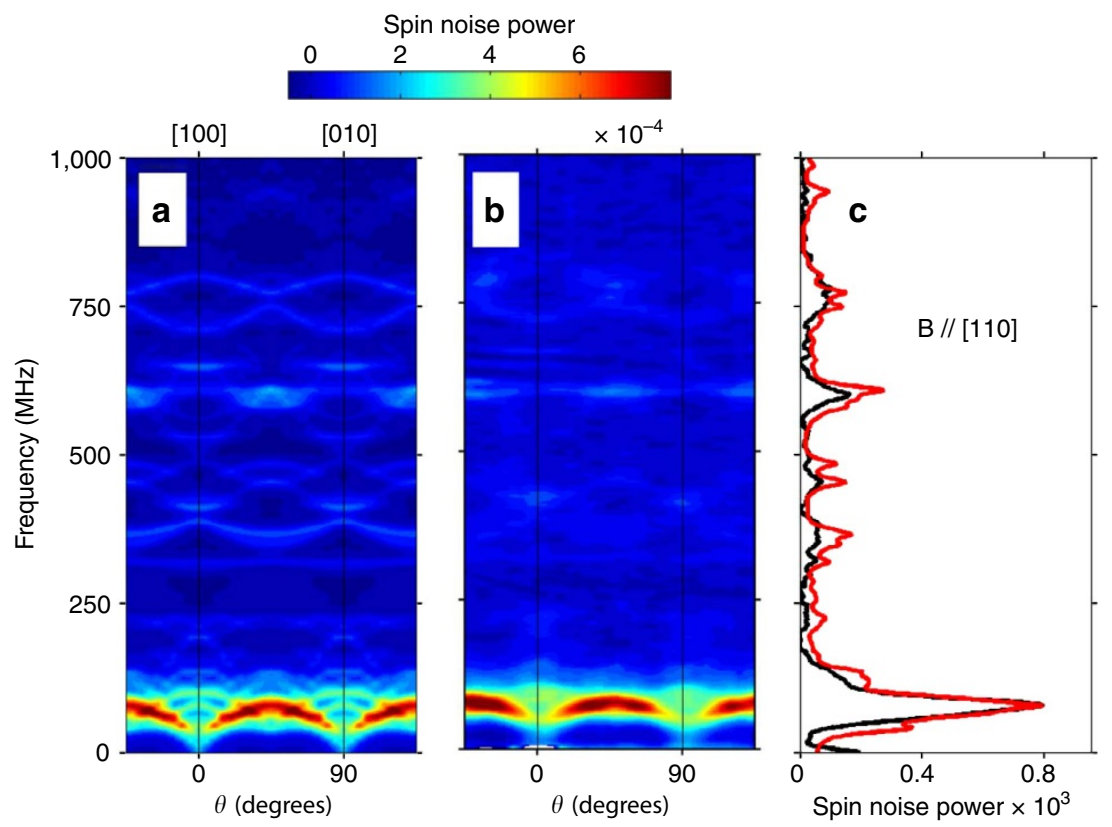

Figure 4 | Angular resolved spin noise of ${ }^{55} \mathbf{M n}$ in the $\mathbf{Q W}$ grown on $\mathbf{C d T e}$ at $\mathbf{B}_{\mathbf{T}}=\mathbf{5} \mathbf{~ m T}$. (a) Contour plot of spin noise spectra calculated for $\gamma_{T}=50 \mathrm{MHz}$, and $D_{0}=-40 \mathrm{neV}$. (b) Experimental contour plot $(T=5 \mathrm{~K})$. (c) Experimental (black line) and calculated spectra (red line) at $\theta=45^{\circ}$ $(B / /[110])$

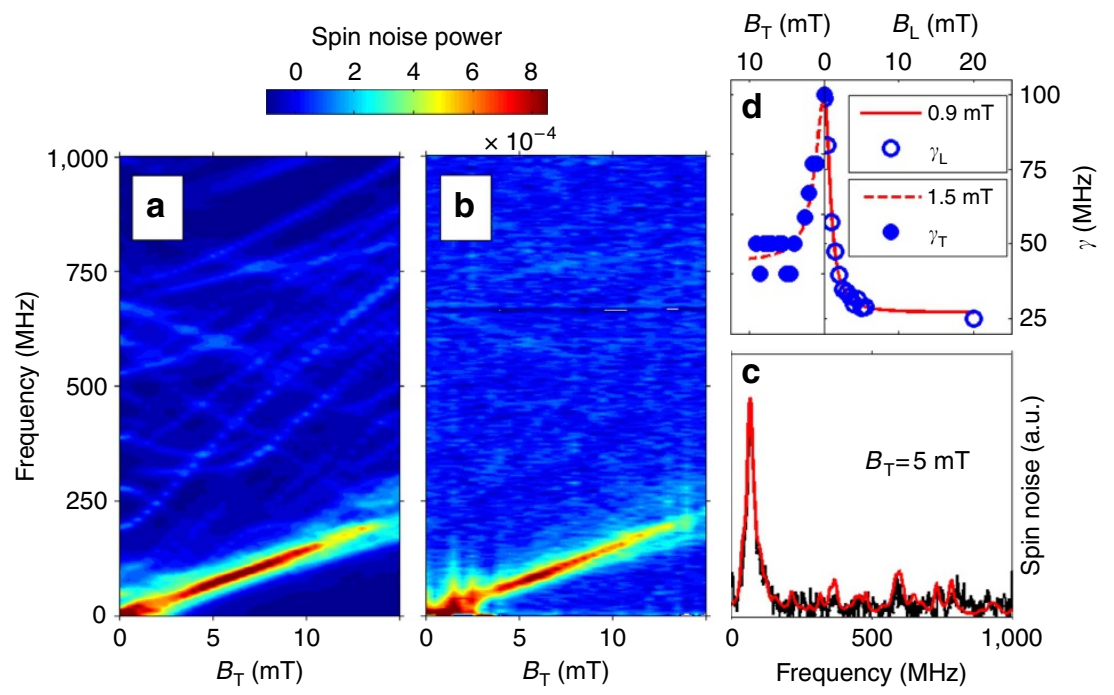

Figure 5 | Spin noise spectra of ${ }^{55} \mathrm{Mn}$ in the QW grown on CdTe versus magnetic field intensity at $\boldsymbol{\theta}=\mathbf{3 0}^{\circ}$. (a) Contour plot of spin noise spectra calculated for $\gamma_{T}=50 \mathrm{MHz}$, and $D_{0}=-40 \mathrm{neV}$. (b) Experimental contour plot $(T=6 \mathrm{~K})$. (c) Fitting equation (2) to the experimental spectrum determines $\gamma$. (d) Obtained values of $\gamma_{T}$ in transverse field and $\gamma_{L}$ in longitudinal fields (spectra in longitudinal fields given in Supplementary Fig. 4). Red curves are fits of $\gamma_{L}$ and $\gamma_{T}$ to lorentzian (HWHM $=0.9$ and $1.5 \mathrm{mT}$, respectively).

with $g_{F}=1$. These lines are spread in frequency both by the crystal field, and by the quadratic Zeeman effect which arises because of the gradual decoupling of electronic and nuclear spins. The quadratic Zeeman effect becomes more visible above $10 \mathrm{mT}$, where the $g_{F}=1$ line progressively broadens due to its splitting in many unresolved individual lines. Barely visible transitions at higher frequencies correspond to inter-hyperfine transitions $\Delta F=1$. We point out that all the peaks of the unconventional spectra revealed by our low-field measurements, must evolve and contribute at high field to the well-known structure of the Mn spin resonance spectra consisting of six equally spaced lines.

Linewidths. Our results give new insights in the Mn spin relaxation mechanisms at low magnetic field, a regime usually not accessible by conventional spin resonance techniques. In the transverse configuration, although the presence of many unresolved lines complicates the determination of the broadening parameter $\gamma_{\mathrm{T}}$, it can be estimated by fitting the experimental spectra with Equation (2) (see Fig. 5c). Fig. 5d shows that $\gamma_{T}$ notably decreases from $100 \mathrm{MHz}$ at zero field to $40 \mathrm{MHz}$ above a characteristic field $B_{\mathrm{c}} \sim 1 \mathrm{mT}$.

A potential source of broadening in this range of $\mathrm{Mn}$ composition $x$, is the dipolar interactions among the ensemble of electronic $\mathrm{Mn}$ spins ${ }^{16}$. Here, a Lorentzian shape is expected, with the wings of the line formed by spins with a first neighbour at short distance, and the centre due to spins with no neighbour in a volume $\sim 1 / x N_{0}$, where $N_{0}=4 / a^{3}$ is the density of cation sites in CdTe, with a cubic lattice parameter $a=0.648 \mathrm{~nm}$. 
Adapting the calculation of moments of ref. 17 to a $\mathrm{Mn}$ spin with $S=5 / 2$, and $g=2$, we obtain an effective field $B_{\text {eff }} \simeq 5 g \mu_{\mathrm{B}} N_{0} x \sqrt{S(S+1)} \mu_{0} / 4 \pi=0.4 \mathrm{mT}$. Assuming that the effect of hyperfine coupling affects the Landé factor and not $B_{\text {eff, }}$, the resulting linewidth with $g=1$ is $\gamma=g \mu_{\mathrm{B}} B_{\text {eff }} / \hbar=35 \mathrm{MHz}$, to be increased by a factor $10 / 3$ at zero field ${ }^{16}$.

In a longitudinal field, $\gamma_{\mathrm{L}}$ is much easier to measure, as all the intra-hyperfine lines merge at zero frequency. Spectra are given in Supplementary Fig. 3. We expect the dipolar broadening to be totally suppressed as soon as the applied field is larger than the dipolar fields. This is the case in Fig. $5 \mathrm{~d}$ where $\gamma_{\mathrm{L}}$ rapidly decreases; however, it saturates at a finite value, suggesting that a relaxation mechanism, with $T_{1}=\gamma_{\mathrm{L}}^{-1}$, remains to be identified.

In both cases, a simple estimation of dipolar broadening qualitatively explains the behaviour of the linewidth upon applying a magnetic field, and gives reasonable orders of magnitude. However, there is another, smaller contribution which appears to be quite independent of the applied field. Estimates of other sources of broadening are given in Supplementary Note 2. Supplementary Fig. 4 shows that the linewidth is robust to changes of temperature and excitation power.

\section{Discussion}

As an outlook, we emphasize that the present measurements can be extended to higher magnetic fields by using large bandwidth spin noise spectroscopy techniques ${ }^{18,19}$. This will permit to study the Paschen-Back regime, when electronic and nuclear spins are completely decoupled. In this regime, because of the hyperfine interaction, the Mn electronic spin precession frequency depends on the orientation of the nuclear spin relative to the applied field ${ }^{20}$. The fluctuation spectra will therefore directly reveal the nuclear spin populations, an information which cannot be easily addressed by other spectroscopic methods.

The sensitivity is high enough to open the way towards the detection of a few spins in systems where the decoherence because of nuclear spins can be suppressed by isotopic enrichment $^{21-23}$, and towards spin resonance microscopy with important applications in biology and materials science ${ }^{24}$. Additional coupling of the spins to a microcavity should enable single spin detection ${ }^{10,25,26}$ : this will open a fascinating opportunity to explore quantum jumps of the Larmor frequency of a single Mn spin (see Supplementary Note 2), and to realize a high-fidelity readout of its nuclear $\operatorname{spin}^{27}$.

\section{Methods}

Quantum well samples. Three QW were grown by molecular beam epitaxy on (100) substrates. A $14 \mathrm{~nm}$ wide $\mathrm{CdTe} / \mathrm{Cd}_{0.75} \mathrm{Mg}_{0.25} \mathrm{Te} \mathrm{QW}$ was grown on $\mathrm{CdTe}$ as a reference: no spin noise signal could be detected for this sample without $\mathrm{Mn}$. Two $\mathrm{Cd}_{1-x} \mathrm{Mn}_{x} \mathrm{Te} / \mathrm{Cd}_{0.75} \mathrm{Mg}_{0.25} \mathrm{Te}$ were grown in the same conditions as the reference QW: a $14 \mathrm{~nm}$ wide QW grown on CdTe and a $20 \mathrm{~nm}$ wide QW grown on $\mathrm{Cd}_{0.96} \mathrm{Zn}_{0.04} \mathrm{Te}$. Using Vegard's law and the lattice parameters of CdTe and $\mathrm{ZnTe}$ (ref. 28) and MnTe (ref. 29), one can expect a shear strain along the growth axis, isotropic in the growth plane, of $2 \times 10^{-5}$, in tension, for the $\mathrm{Cd}_{1-x} \mathrm{Mn}_{x} \mathrm{Te}$ QW grown on CdTe and $-2 \times 10^{-3}$, in compression, for the one grown on $\mathrm{Cd}_{0.96} \mathrm{Zn}_{0.04} \mathrm{Te}$. The Mn compositions, $x=0.001$, have been controlled by magneto-reflectivity. Photoluminescence revealed a trion line, likely due to residual or photogenerated holes in the QWs.

Experimental setup. We developed a specific setup adapted to Mn spin noise spectroscopy, which requires a large bandwidth up to $1 \mathrm{GHz}$, and a high sensitivity. To that purpose we used an avalanche diode with a very low noise equivalent power (typical NEP $0.4 \mathrm{pW} / \mathrm{Hz}^{1 / 2}$ ), and a short response time of $0.5 \mathrm{~ns}$. The sensitivity is then maximized by detecting the spin fluctuations in nearly crossed polarization (average power on the detector is less than $\sim 1 \mu \mathrm{W}$ ), while keeping a relatively high probe power on the sample (typically $\sim 1 \mathrm{~mW}$ in our experiments) ${ }^{30}$. In these conditions, the attenuated laser is at the shot noise. Subtraction of the photon noise from the total noise is achieved by alternatively measuring the power spectra of the signal and reference beams (see Fig. 1a). The normalized spin noise power in units of the photon noise, and corrected from the apparatus response function is then given by $S_{\text {spin }}(f)=\frac{S_{\text {signal }}(f)-S_{\text {ref }}(f)}{S_{\text {signal }}(f)+S_{\text {ref }}(f)}$. The samples are mounted on the cold finger of a helium cryostat, and placed at the centre of two-axis Helmholtz coils. One narrowband continuous-wave diode laser (from Toptica) is tuned to the excitonic resonance of the bulk or of the QWs (see Supplementary Fig. 5). With a laser spot size $\sim 5 \mu \mathrm{m}\left(\sim 4 \times 10^{6} \mathrm{Mn}\right.$ atoms lie within the spot size in the case of the QW). The spin noise signal is continuously digitized at $2 \mathrm{GHz}$ and processed by a field-programmable gate array (Agilent card U1080A), to obtain the spin noise power spectrum. Typically, each spectrum requires a few minutes of signal averaging.

\section{References}

1. Aleksandrov, E. B. \& Zapassky, V. S. Magnetic resonance in the Faraday-rotation noise spectrum. Zh. Eksp. Teor. Fiz. 81, 132-138 (1981).

2. Crooker, S. A., Rickel, D. G., Balatsky, A. V. \& Smith, D. L. Spectroscopy of spontaneous spin noise as a probe of spin dynamics and magnetic resonance. Nature 431, 49-52 (2004).

3. Kuzmich, A., Bigelow, N. P. \& Mandel, L. Atomic quantum non-demolition measurements and squeezing. Europhys. Lett. 42, 481 (1998).

4. Geremia, J., Stockton, J. K. \& Mabuchi, H. Real-time quantum feedback control of atomic spin-squeezing. Science 304, 270-273 (2004).

5. Julsgaard, B., Kozhekin, A. \& Polzik, E. S. Experimental long-lived entanglement of two macroscopic objects. Nature 413, 400-403 (2001).

6. Crooker, S. et al. Spin noise of electrons and holes in self-assembled quantum dots. Phys. Rev. Lett. 104, 036601 (2010).

7. Li, Y. et al. Intrinsic spin fluctuations reveal the dynamical response function of holes coupled to nuclear spin baths in (In,Ga)As quantum dots. Phys. Rev. Lett. 108, 186603 (2012).

8. Horn, H. et al. Spin noise spectroscopy of donor-bound electrons in $\mathrm{ZnO}$. Phys. Rev. B 87, 045312 (2013).

9. Zapasskii, V. S. et al. Optical spectroscopy of spin noise. Phys. Rev. Lett. 110, 176601 (2013).

10. Dahbashi, R., Hubner, J., Berski, F., Pierz, K. \& Oestreich, M. Optical spin noise of a single hole spin localized in an (InGa)As quantum dot. Phys. Rev. Lett. 112, 156601 (2014).

11. Poltavtsev, S. V. et al. Spin noise spectroscopy of a single quantum well microcavity. Phys. Rev. B 89, 081304(R) (2014).

12. Yang, L. et al. Two-colour spin noise spectroscopy and fluctuation correlations reveal homogeneous linewidths within quantum-dot ensembles. Nat. Commun 5, 4949 (2014).

13. Abragam, A. Principles of Nuclear Magnetism (Oxford University Press, 1961)

14. Causa, M., Tovar, M., Oseroff, S. B., Calvo, R. \& Giriat, W. Spin-lattice coefficients of $\mathrm{Mn}^{2+}$ in II-VI compounds. Phys. Lett. A 77, 473-475 (1980).

15. Landau, L. D. \& Lifshitz, E. M. Statistical Physics (Pergamon Press, 1980).

16. Abragam, A. \& Bleaney, B. Electron Paramagnetic Resonance of Transition Ions (Clarendon Press, 1970).

17. Kittel, C. \& Abrahams, E. Dipolar broadening of magnetic resonance lines in magnetically diluted crystals. Phys. Rev. 90, 238-239 (1953).

18. Müller, G. M., Römer, M., Hübner, J. \& Oestreich, M. Gigahertz spin noise spectroscopy in n-doped bulk GaAs. Phys. Rev. B 81, 121202(R) (2010).

19. Hübner, J. et al. Rapid scanning of spin noise with two free running ultrafast oscillators. Opt. Express 21, 5872-5878 (2013).

20. Cronenberger et al. Optical pump-probe detection of manganese hyperfine beats in (Cd,Mn)Te crystals. Phys. Rev. Lett. 110, 077403 (2013).

21. Balasubramanian, G. et al. Ultralong spin coherence time in isotopically engineered diamond. Nat. Mater 8, 383-387 (2009).

22. Tyryshkin, A. M. et al. Electron spin coherence exceeding seconds in high-purity silicon. Nat. Mater 11, 143-147 (2011).

23. Sleiter, D. J. et al. Optical pumping of a single electron spin bound to a fluorine donor in a ZnSe nanostructure. Nano Lett. 13, 116-120 (2013).

24. Blank, A., Dunnam, C. R., Borbat, P. P. \& Freed, J. H. High resolution electron spin resonance microscopy. J. Magn. Reson. 165, 116-127 (2003).

25. Salis, G. \& Moser, M. Faraday-rotation spectrum of electron spins in microcavity-embedded GaAs quantum wells. Phys. Rev. B 72, 115325 (2005).

26. Hu, C. Y., Young, A., O’Brien, J. L., Munro, W. J. \& Rarity, J. G. Giant optical Faraday rotation induced by a single-electron spin in a quantum dot: applications to entangling remote spins via a single photon. Phys. Rev. B 78, 085307 (2008)

27. Pla, J. J. et al. High-fidelity readout and control of a nuclear spin qubit in silicon. Nature 496, 334-338 (2013).

28. Hans Landolt, H. et al. in Group 3, Crystal and solid state physics. Semiconductors Vol. 17 (eds Madelung, O., Schulz, M. \& Weiss, H.) (Springer-Verlag, 1982).

29. Janik, E. et al. Structural properties of cubic MnTe layers grown by MBE. Thin Solid Films 267, 74-78 (1995).

30. Glasenapp, P. et al. Resources of polarimetric sensitivity in spin noise spectroscopy. Phys. Rev. B 88, 165314 (2013). 


\section{Acknowledgements}

D.S. and S.C warmly acknowledge stimulating discussions with M. Dyakonov and M. Myara. This work was supported by the French ANR research project SNS (Grant no. 2011-BS04-018 01).

\section{Author contributions}

D.S. supervised the whole project. D.S. and S.C. conceived the experiment, built the experimental apparatus and analysed the data. D.S. wrote the manuscript in close collaboration with all authors, particularly J.C. H.B grew the samples. D.F. performed the magneto-optical spectroscopy of the quantum wells. All authors contributed to numerous discussions and revised the manuscript.

\section{Additional information}

Supplementary Information accompanies this paper at http://www.nature.com/ naturecommunications
Competing financial interests: The authors declare no competing financial interests.

Reprints and permission information is available online at http://npg.nature.com/ reprintsandpermissions/

How to cite this article: Cronenberger, S. et al. Atomic-like spin noise in solid state demonstrated with manganese in cadmium telluride. Nat. Commun. 6:8121 doi: 10.1038/ncomms9121 (2015).

\section{(c) (i)}

This work is licensed under a Creative Commons Attribution 4.0 International License. The images or other third party material in this article are included in the article's Creative Commons license, unless indicated otherwise in the credit line; if the material is not included under the Creative Commons license, users will need to obtain permission from the license holder to reproduce the material. To view a copy of this license, visit http://creativecommons.org/licenses/by/4.0/ 